\title{
Effect of Er:YAG Laser on Surface Properties, Bacterial and Lipopolysaccharide Clearance, and Human Gingival Fibroblast Adhesion: An In-Vitro Peri-Implantitis Model
}

\section{Cheng-Ying Wang}

National Taiwan University Hospital

\section{Bor-Shiunn Lee}

National Taiwan University Hospital

Ya-Ting Jhang

National Taiwan University Hospital

Kevin Sheng-Kai Ma

National Taiwan University

Chen-Pang Huang

National Taiwan University Hospital

Kuan-Lun Fu

National Taiwan University Hospital

Chern-Hsiung Lai

Kaohsiung Medical University

Mark Yen-Ping Kuo

National Taiwan University Hospital

Yi-Wen Chen ( $\sim$ yiwenchen@ntu.edu.tw )

National Taiwan University Hospital

\section{Research Article}

Keywords: lipopolysaccharide (LPS), Porphyromonas gingivalis, chlorhexidine, fluorescent microscopy.

Posted Date: August 19th, 2021

DOl: https://doi.org/10.21203/rs.3.rs-812111/v1

License: (1) This work is licensed under a Creative Commons Attribution 4.0 International License.

Read Full License 


\section{Abstract}

Purpose: To investigate the effect of Er:YAG laser treatment on lipopolysaccharide (LPS) clearance and fibroblast adhesion in an ex vivo peri-implantitis model.

Methods: Grade IV titanium discs $(n=216)$ were used and allocated to 6 groups. Group 1 was the negative control without Porphyromonas gingivalis inoculation. Discs in Groups 2 to 6 were incubated with $P$. gingivalis to form a biofilm. Group 3 received $0.12 \%$ chlorhexidine irrigation and Group 4 received titanium curettage to remove the biofilm. Group 5 was treated with Er:YAG laser irradiation and Group 6 was treated with titanium curettage plus Er:YAG laser irradiation. The contact angle and surface roughness were measured after the various treatments. The surface microstructure and residual bacteria were examined using scanning electron microscopy and confocal laser scanning microscopy, respectively. Residual LPS was examined using a limulus amoebocyte lysate assay and human gingival fibroblast adhesion was quantified using fluorescent microscopy.

Results: Curettage plus Er:YAG laser irradiation was the most effective method for removing bacteria and LPS. No significant difference in the amount of fibroblast adhesion was found between the control and Group 6.

Conclusion: Combined use of Er:YAG laser irradiation and curettage is a promising therapy for managing peri-implantitis .

\section{Introdution}

Peri-implantitis is characterized by progressive loss of supporting bone and inflammation of peri-implant connective tissue ${ }^{1}$. The patient- and implant-level prevalence of peri-implantitis has been reported to be approximately $22-45 \%$ and $11.4 \%$, respectively ${ }^{2-4}$. The principal etiology of peri-implantitis involves bacterial biofilm accumulation ${ }^{1,5,6}$, in which pathogenic species including Porphyromonas gingivalis ${ }^{7}$ and virulence factors such as lipopolysaccharides (LPS) ${ }^{8}$ have been identified. Hence, the primary goal of peri-implantitis management is to remove the biofilm and LPS from implant surfaces to resolve soft tissue inflammation and prevent further bone loss.

Mechanical, non-surgical therapy for peri-implantitis lesions remains unpredictable, with clinical benefits limited to 6 to 12 months $^{9}$, and can alter the microstructure of the implant surface ${ }^{10}$. An alternative treatment for peri-implantitis is laser therapy. Among various minimally invasive lasers ${ }^{11-13}$, the erbiumdoped yttrium aluminum garnet (Er:YAG) laser has been frequently employed for peri-implantitis management ${ }^{11-14}$. In addition to its ability to ablate hard tissue ${ }^{15,16}$, the wavelength of the Er:YAG laser is approximate to the absorption wavelength of LPS, which enables the Er:YAG laser to exert a bactericidal effect on implant surfaces ${ }^{17-22}$. Moreover, Er:YAG laser treatment of titanium implants creates a surface with appropriate wettability ${ }^{18,23}$ that favors the adhesion of epithelial cells, gingival 
fibroblasts, and osteoblast-like cells ${ }^{21}$. Furthermore, Er:YAG laser irradiation is effective for the debridement and removal of inflamed granulomatous tissue from peri-implant defects ${ }^{1}$.

Previous in vitro studies have demonstrated the efficacy of Er:YAG laser treatment in the ablation of bacterial biofilms or calculus ${ }^{21,24}$. However, residual bacteria and LPS after Er:YAG laser ablation might impede the attachment of human gingival fibroblasts (HGFs) and this topic has not been studied. We conducted this study to address the effect of Er:YAG laser treatment on residual bacteria and LPS in an in vitro titanium-based peri-implantitis model.

\section{Results}

\section{Wettability measurement of the titanium surface}

The contact angle of pure titanium plates (Group 1) was significantly higher than those of titanium discs with the $P$. gingivalis biofilm, $0.12 \% \mathrm{CHX}$ washing, and curettage $(p<0.0001, p<0.001, p<0.05)$. For Er:YAG laser treatment alone, or the combined use of curettage and Er:YAG laser treatment, the contact angle was not significantly different from that of the control group (Fig. 1).

\section{Surface roughness analysis and measurement}

Surface roughness indicated the influence of the biofilm, $\mathrm{CHX}$ washing, curettage, laser treatment, and the combination of laser treatment and curettage on the surface structure of the titanium plates. No significant differences were found among the groups (Fig. 2). These findings suggested that neither mechanical debridement nor Er:YAG irradiation altered Ra.

\section{SEM findings of the titanium surface following debridement}

No bacteria were observed on the titanium surface in the control group (Fig. 3). By contrast, a P. gingivalis biofilm was prominently observed in Group 2 . After $0.12 \% \mathrm{CHX}$ washing (Group 3), P. gingivalis clusters were still visible on the titanium surface. Similarly, titanium debris embedded with bacterial residue was observed after curettage plus PBS washing (Group 4). These findings indicated that curettage alone was insufficient to remove all bacteria. By contrast, Er:YAG laser debridement (Group 5) was effective at removing $P$. gingivalis and the surface clearance was similar to that of the control group. Curettage followed by Er:YAG laser treatment (Group 6) was also effective at removing $P$. gingivalis. However, horizontal and vertical scratches caused by curettage were observed.

\section{Live and dead bacteria on the titanium surface under confocal microscopy}

SYTO 9 and propidium iodide were used to distinguish live and dead bacteria based on their different abilities to penetrate the cell membrane (Fig. 4(a)). The control group (Group 1) did not produce fluorescent signals because it did not contain bacteria. The fluorescence intensity of $P$. gingivalis in Group 2 was the highest among the 6 groups. Bacterial counts decreased after $0.12 \% \mathrm{CHX}$ washing (Group 3 ) but bacteria were not completely removed. Curettage (Group 4) eliminated most bacteria, resulting in 
fewer fluorescent signals compared with Group 3. Laser irradiation (Group 5) and combined use of curettage and laser irradiation (Group 6) resulted in even fewer fluorescent signals, with only a few scattered red signals being observed. Quantitative analysis of fluorescence intensity using Zen software revealed that Group 2 had the highest fluorescence intensity (Fig. 4(b)(c)), followed by Group 3 (0.12\% CHX washing) and Group 4 (curettage). Groups 5 and 6 had the lowest fluorescence intensity and these results were consistent with the SEM findings.

\section{Kinetic turbidimetric assay for residual endotoxins}

After immersion of the titanium discs in endotoxin-free LAL water for $24 \mathrm{~h}$, the absorbance in the kinetic turbidimetric assay for titanium discs was measured (Fig. 5). The absorbance of titanium discs with $P$. gingivalis biofilm formation (Group 2) was $0.679 \pm 0.009$, which was the highest among the 6 groups. After $0.12 \% \mathrm{CHX}$ washing (Group 3), the absorbance was significantly lower than that of Group 2 ( $p<$ 0.05 ). The absorbance after curettage (Group 4) was not significantly different from that of Group 2 ( $p=$ 0.078). After Er:YAG laser irradiation and after combined use of curettage and Er:YAG laser irradiation, the absorbance was significantly lower than that of Group $2(p<0.0001, p<0.0001$, respectively). These findings suggest that Er:YAG laser treatment was able to reduce residual endotoxins on the titanium surface, such as LPS.

\section{Quantitative evaluation of HGF adhesion after different surface treatments}

Evaluation of HGF adhesion (per square millimeter) after different surface treatments was quantified using fluorescent microscopy. The results showed that the HGF adhesion in Group 2 was the lowest among the 6 groups (Fig. 6). In addition, combined use of curettage and Er:YAG laser irradiation (Group 6) resulted in the highest HGF adhesion, followed by curettage (Group 4) or Er:YAG laser treatment (Group 5) alone. The titanium discs treated using $0.12 \% \mathrm{CHX}$ washing (Group 3 ) had the most unfavorable surface in terms of HGF adhesion.

\section{Discussion}

This study investigated HGF adhesion to $P$. gingivalis biofilm-contaminated titanium discs that were treated with different clinically used methods for peri-implantitis, including $\mathrm{CHX}$ washing, mechanical debridement, and Er:YAG laser irradiation. The findings indicated that combined use of curettage and Er:YAG laser irradiation was the most effective at removing bacteria and residual endotoxins, and achieved a beneficial microenvironment for HGF attachment to the titanium surface.

The surface roughness and contact angle of a titanium plate after a designated treatment has been shown to be associated with cell adhesion ${ }^{25-27}$. In this study, SEM revealed that mechanical debridement using curettage created scratches on the titanium surface (Fig. 3), although the roughness was not significantly changed (Fig. 2). Regarding water-surface interactions, a large water contact angle indicates hydrophobicity, whereas a small water contact angle suggests hydrophilicity ${ }^{28-30}$. Moreover, contact angles of less than $10^{\circ}$ are considered super hydrophilic, those between $10^{\circ}$ and $90^{\circ}$ are 
considered hydrophilic, those between $90^{\circ}$ and $120^{\circ}$ are considered hydrophobic, and those over $120^{\circ}$ are considered superhydrophobic ${ }^{28-30}$. The contact angles of all groups in this study were less than $90^{\circ}$, indicating that the surfaces were hydrophilic. Specifically, the contact angles of Groups 2 to 6 were all significantly less than that of Group 1, which suggested that inoculation with bacteria and treatment including $0.12 \% \mathrm{CHX}$ washing, titanium curettage, and laser treatment all altered surface hydrophilicity. The results may be attributable to residual endotoxins, such as LPS, and $P$. gingivalis on the titanium plates rendering the surfaces hydrophilic ${ }^{31,32}$. In addition, the contact angles of both Group 5 (Er:YAG laser treatment) and Group 6 (combined use of curettage and Er:YAG laser treatment) were higher than those of Groups 2-4. These findings were consistent with Group 6 demonstrating the most effective removal of bacteria and LPS (Figs. 4 and 5), causing similar HGF cell attachment to that of Group 1.

Group 2 exhibited the highest fluorescence intensity under confocal microscopy (Fig. 4) and SEM examination also showed a $P$. gingivalis colony $2-3 \mu \mathrm{m}$ in size. Therefore, no HGF adhesion was observed at 24,72 , and $150 \mathrm{~h}$, suggesting that $P$. gingivalis had an adverse effect on HGF cell adhesion and proliferation. This adverse bacterial effect on cell adhesion has been suggested to result from alteration of the extracellular matrix and its components ${ }^{33-36}$. Propidium iodide signals, which represented dead bacteria, were more intense than SYTO 9 signals, which represented live bacteria in all groups (Fig. 4). Residual bacterial contamination was more evident for $0.12 \% \mathrm{CHX}$ treatment, as evidenced by higher fluorescence intensity, compared with mechanical debridement via titanium curettage, indicating that the latter was more effective at removing bacteria. In addition, Group 3 displayed a low density of nuclei characterized by DAPI, without any cytoskeletal signal under fluorescent microscopy (Fig. 6). Both the low cell count and the lack of cytoskeletal signals suggested poor HGF adhesion, which is in line with previous studies ${ }^{37}$. The reason was ascribed to the limited bactericidal effect of $0.12 \% \mathrm{CHX}$ and the toxicity of $0.12 \% \mathrm{CHX}$ to HGF. A previous in vitro study showed that $\mathrm{CHX}$ causes bacterial lysis by destroying the cell walls and inhibiting fibroblast attachment ${ }^{38}$. The bactericidal effect of Er:YAG laser irradiation combined with titanium curettage (Group 6) was superior to curettage alone (Group 4). The findings are in accordance with a previous study demonstrating that the bactericidal effect of Er:YAG laser irradiation on $P$. gingivalis is stronger than that of titanium curettage ${ }^{21}$. The amount of HGF attachment in the Ti curettage group was also lower than that of the Er:YAG laser irradiation group. The reason was ascribed to the greater amount of residual LPS after curettage than after laser treatment. The other possible reason was that curettage created debris on the surface of the titanium discs. The fluorescence signal intensity and LPS removal ability did not differ significantly between Er:YAG laser treatment with (Group 6) and without (Group 5) curettage. Nevertheless, Group 6 exhibited more HGF attachment than Group 5 and did not differ significantly from the control group. Previous studies have elucidated the utility of Er:YAG laser treatment in the clearance of bacterial biofilms 21,24 , but the influence of residual bacteria and LPS after Er:YAG laser ablation on the attachment of HGFs has not been studied. LPS is released from the cell walls of gram-negative bacteria and can cause inflammation and toxicity to the host ${ }^{8}$. HGF attachment plays a critical role in the successful integration of gingival soft tissue and titanium implants ${ }^{39}$. The removal of LPS and HGF attachment achieved by 
laser irradiation plus curettage indicated the potential clinical efficacy for gingival fiber attachment to implant surfaces, which is crucial for forming a barrier against exposure to periodontal pathogens 40,41 .

This study used Grade 4 titanium plates $(\mathrm{Ra}=1.3 \mu \mathrm{m})$ but the roughness of commercialized implants is approximately 1 to $3 \mu \mathrm{m}$, and the implant macro design, surface morphology, thread geometry, and thread pitch are more complicated, which has been shown to affect osseointegration ${ }^{42}$. Different roughnesses and complicated surface structures can influence the efficiency of surface treatments for implants 43,44 . In addition, peri-implantitis is typically accompanied by intrabony defects. The field of view is limited in clinical practice compared to in vitro models; both curettage and laser treatment are more feasible for achieving optimized bactericidal activity in an in vitro study. Furthermore, peri-implantitis is associated with complex bacterial species, and $P$. gingivalis is only one of the predominant bacteria ${ }^{1}$. These are all limitations in determining the actual efficacy of Er:YAG laser with curettage for treating peri-implantitis. In summary, this study was conducted with the aim of providing an optimal strategy for peri-implantitis management. The findings support the use of Er:YAG laser treatment with curettage to reduce bacteria and residual endotoxins, and optimize HGF attachment to implants, thereby achieving a beneficial periimplant microenvironment for implant maintenance. Future studies could focus on identifying osteoblast adhesion on laser-treated titanium plates, as well as the osteogenic factors that underlie the process. The effect of laser treatment on the microenvironment of periodontium with peri-implantitis also requires further study.

\section{Methods}

\section{Preparation of titanium samples}

Titanium discs were prepared as described previously ${ }^{45}$. Briefly, 216 commercially pure Grade 4 titanium discs ( $\varnothing=15 \mathrm{~mm}$, thickness: $2 \mathrm{~mm}$, surface roughness: $\mathrm{Ra}=1.3 \mu \mathrm{m}$; Ultimate Materials Technology, Hsinchu, Taiwan) were used. After being washed with distilled water and acetone 3 times in a Transsonic ultrasonic bath (Elma Ultrasonic, Singen, Germany), the titanium discs were autoclaved $\left(121^{\circ} \mathrm{C}, 2 \mathrm{~atm}, 30\right.$ min) and dried using laminar flow with UV irradiation overnight.

\section{P. gingivalis inoculation and surface treatments of the titanium discs}

The bacterial inoculation was performed as follows. P. gingivalis $\left(\operatorname{ATCC}^{\circledR} 33277^{\mathrm{TM}}\right)$ were cultured in a brain heart infusion broth (BD Bacto, REF 237500) supplemented with yeast extract, L-cysteine hydrochloride, and resazurin. Subsequently, P. gingivalis with optical density $=0.1$ at $600 \mathrm{~nm}$ was inoculated on the titanium discs and then placed in a 24-well plate and cultured under anaerobic conditions for $72 \mathrm{~h}$ to allow biofilm formation.

The sterilized titanium discs were divided into 6 groups (Fig. 7). Group 1 did not receive bacterial inoculation or debridement and served as a control. Group 2 received only $P$. gingivalis inoculation without further treatment. Group 3 received $P$. gingivalis inoculation followed by 
$0.12 \%$ chlorhexidine $(\mathrm{CHX})$ irrigation $(10 \mathrm{~mL}$ each time, $30 \mathrm{~mL}$ in total). Group 4 received $P$. gingivalis inoculation followed by periodontal titanium curette ( $8 \mathrm{~mm}$ in diameter, Langer $1 / 2$, item code: 7103, Kohler Medizintechnik, GmbH \& Co, Ltd, Stockach, Germany) debridement (30 vertical strokes with hand pressure for each disc) and normal saline irrigation ( 5 times per 10 strokes, $1 \mathrm{~mL}$ each time). Group 5 received $P$. gingivalis inoculation followed by Er:YAG laser (AdvErL Evo, Morita Corporation, Tokyo, Japan) irradiation using a C600F tip (600 $\mu \mathrm{m}$ in diameter, item code: no. 34-8001703, Morita Corporation) with water cooling at a pulse energy of $80 \mathrm{~mJ}$ and frequency of 25 pulses per second after inoculation. The total irradiation time was $10 \mathrm{~min}$ and the energy density was $3.87 \mathrm{~J} / \mathrm{cm}^{2}$. Group 6 received $P$. gingivalis inoculation followed by curette debridement and Er:YAG laser irradiation (combining the treatments of Groups 4 and 5).

\section{Contact angle measurement}

The surface hydrophilicity of the titanium discs after surface treatment was determined by measuring the contact angle $(n=6)$ with one drop $(0.5 \mu \mathrm{L})$ of deionized water using a contact angle goniometer (FTA125; First Ten Ångstroms, Portsmouth, VA, USA). Three independent measurements were performed for each sample.

\section{Surface roughness measurement}

To evaluate the surface roughness of the titanium discs, a Surfcorder ET 200 profilometer (Kosaka, Tokyo, Japan) was used $(n=6)$. The tracing diamond tip was $2 \mu \mathrm{m}$ with a tracing length of $4 \mathrm{~mm}$, force of $200 \mu \mathrm{N}$, tracing speed of $0.2 \mathrm{~m} / \mathrm{s}$, and cutoff value of $0.8 \mathrm{~mm}$. Six tracings were performed at different locations on the surface of each specimen. The average surface roughnesses were calculated as $\mathrm{Ra}$ values.

\section{Surface morphology observation using scanning electron microscopy}

The titanium discs $(n=6)$ were washed with phosphate-buffered saline (PBS) twice and dehydrated with serial ethanol ( $50 \%, 70 \%, 80 \%, 90 \%, 95 \%, 99 \%$, for 15 min each) after surface treatments. After dehydration, the samples were dried using a critical point dryer, mounted on aluminum stubs, sputtercoated with approximately 20-nm-thick gold/palladium, and finally examined using field-emission scanning electron microscopy (FE-SEM; Nova NanoSEM 230, FEI Co, Brno, Czech Republic) with an accelerating voltage of $15 \mathrm{kV}$.

\section{Detection and quantification of adherent bacteria}

The double staining of SYTO 9/PI has been used to quantify viable and dead bacteria. In the present study, adherent bacteria were examined and quantified using the SYTO 9/PI staining technique (LIVE/DEAD BacLight Bacterial Viability Kit; Invitrogen, Carlsbad, CA, USA). After fixation in 4\% paraformaldehyde for 10 min, SYTO 9/PI double staining was performed on the titanium discs $(n=6)$ at $37^{\circ} \mathrm{C}$ in the dark, followed by incubation for $15 \mathrm{~min}$. The samples were then examined using fluorescence 
microscopy (Zeiss LSM780 confocal microscope; Zeiss, Oberkochen, Germany). Three independent measurements were performed for each sample.

\section{Quantification of endotoxin residue on the titanium discs}

The remaining LPS on the titanium discs $(n=6)$ after different surface treatments was measured using a toxin sensor chromogenic limulus amebocyte lysate (LAL) endotoxin assay kit (GenScript, Piscataway, $\mathrm{NJ}$, USA). LAL reagents were added to vials containing the treated titanium discs and an endotoxin standard. After incubation at $37^{\circ} \mathrm{C}$ for $45 \mathrm{~min}$, a chromogenic substrate solution $(100 \mu \mathrm{L})$ was added and incubated at $37^{\circ} \mathrm{C}$ for $6 \mathrm{~min}$. A stop solution $(500 \mu \mathrm{L})$ and color stabilizer $(500 \mu \mathrm{L})$ were added and measured at $545 \mathrm{~nm}$ using a spectrophotometer.

\section{HGF adhesion assay}

Gingival tissues were harvested from the maxillary tuberosity of healthy human donors. Ethical approval and informed consent were obtained from all volunteers (IRB NTUH-REC No. 202002055RIND). All methods were performed in accordance with the relevant guidelines and regulations. HGF were seeded on the surface of the treated titanium discs $(n=6)$ in 24-well plates at a density of $2.5 \times 10^{4}$ cells per disc and cultured for 24,72 , and $120 \mathrm{~h}$ for the adhesion assay. To verify and count the cell numbers, ActinGreen $^{\top m} 488$ (Invitrogen) to indicate a cytoskeleton and 4',6-diamidino-2-phenylindole (DAPI) labeled nucleic acids were used. At each time point, the titanium discs were washed with PBS to remove nonattached cells and fixed in $4 \%$ paraformaldehyde for 10 min followed by ActinGreen ${ }^{\mathrm{Tm}} 488$ and DAPI. Three fields of view per sample were captured with a Zeiss LSM780 confocal microscope (Zeiss). The cell density (cells $/ \mathrm{mm}^{2}$ ) of HGFs was determined using ZEN Offline (Zeiss). Three independent measurements were performed for each sample.

\section{Statistical analysis}

Differences between the control and experimental groups were analyzed using a one-way analysis of variance followed by Tukey's honest significant difference test for multiple comparisons. All data are presented as means \pm standard deviation. Results with $p$-values $<0.05$ were considered to be significant.

\section{Declarations}

\section{Acknowledgements}

This study was supported by grants (106-2314-B-002-035, 109-2314-B-002-032-MY3) from the Ministry of Science and Technology of Taiwan and grants (107-S3852, 109-S4684) from National Taiwan University Hospital.

\section{Author contributions}


Conceptualization, CYW, BSL, YPK and YWC; methodology, CYW, BSL, YTJ, KLF and YWC; formal analysis, YTJ, SKM, CPH and KLF; writing-original draft preparation, CYW, BSL, SKM, CPH and YWC; writing-review and editing, CHL, YPK and YWC; supervision, CYW and YWC; funding acquisition, BSL and YWC. All authors have read and approved the published version of the manuscript.

\section{Competing interests}

The authors declare that they have no conflicts of interest.

\section{Data availability}

The data used to support the findings of this study are available from the corresponding author upon request.

\section{References}

1. Schwarz, F., Derks, J., Monje, A. \& Wang, H.L. Peri-implantitis. J Clin Periodonto/45 Suppl 20, S246S266 (2018).

2. Koldsland, O.C., Scheie, A.A. \& Aass, A.M. Prevalence of peri-implantitis related to severity of the disease with different degrees of bone loss. J Periodonto/81, 231-238 (2010).

3. Derks, J. \& Tomasi, C. Peri-implant health and disease. A systematic review of current epidemiology. J Clin Periodonto/42 Suppl 16, S158-171 (2015).

4. Derks, J. et al. Peri-implantitis - onset and pattern of progression. J Clin Periodonto/43, 383-388 (2016).

5. Sarmiento, H.L., Norton, M.R. \& Fiorellini, J.P. A Classification System for Peri-implant Diseases and Conditions. Int J Periodontics Restorative Dent36, 699-705 (2016).

6. Ogata, Y. Prevalence and Etiology for Peri-implant Diseases. Risk Factors for Peri-implant Diseases (2020).

7. Eick, S. et al. Microbiota at teeth and implants in partially edentulous patients. A 10-year retrospective study. Clin Oral Implants Res27, 218-225 (2016).

8. Ogawa, T. \& Yagi, T. Bioactive mechanism of Porphyromonas gingivalis lipid A. Periodontol 200054, 71-77 (2010).

9. Renvert, S., Roos-Jansaker, A.M. \& Claffey, N. Non-surgical treatment of peri-implant mucositis and peri-implantitis: a literature review. J Clin Periodonto/35, 305-315 (2008).

10. Suarez, F., Monje, A., Galindo-Moreno, P. \& Wang, H.L. Implant surface detoxification: a comprehensive review. Implant Dent22, 465-473 (2013).

11. Kotsakis, G.A., Konstantinidis, I., Karoussis, I.K., Ma, X. \& Chu, H. Systematic review and metaanalysis of the effect of various laser wavelengths in the treatment of peri-implantitis. $J$ Periodonto/85, 1203-1213 (2014). 
12. Mailoa, J., Lin, G.H., Chan, H.L., MacEachern, M. \& Wang, H.L. Clinical outcomes of using lasers for peri-implantitis surface detoxification: a systematic review and meta-analysis. $J$ Periodonto/85, 11941202 (2014).

13. Natto, Z.S., Aladmawy, M., Levi, P.A., Jr. \& Wang, H.L. Comparison of the efficacy of different types of lasers for the treatment of peri-implantitis: a systematic review. Int J Oral Maxillofac Implants30, 338-345 (2015).

14. Swider, K., Dominiak, M., Grzech-Lesniak, K. \& Matys, J. Effect of Different Laser Wavelengths on Periodontopathogens in Peri-Implantitis: A Review of In Vivo Studies. Microorganisms7 (2019).

15. Seka, W.D., Featherstone, J., Fried, D., Visuri, S. \& Walsh, J. Laser ablation of dental hard tissue: from explosive ablation to plasma-mediated ablation, Vol. 2672. (SPIE, 1996).

16. Aoki, A. et al. Periodontal and peri-implant wound healing following laser therapy. Periodontol 200068, 217-269 (2015).

17. Friedmann, A., Antic, L., Bernimoulin, J.P. \& Purucker, P. In vitro attachment of osteoblasts on contaminated rough titanium surfaces treated by Er:YAG laser. J Biomed Mater Res A79, 53-60 (2006).

18. Yamamoto, A. \& Tanabe, T. Treatment of peri-implantitis around TiUnite-surface implants using Er:YAG laser microexplosions. Int J Periodontics Restorative Dent33, 21-30 (2013).

19. Nevins, M. et al. Use of Er:YAG laser to decontaminate infected dental implant surface in preparation for reestablishment of bone-to-implant contact. Int J Periodontics Restorative Dent34, 461-466 (2014).

20. Yoshino, T., Yamamoto, A. \& Ono, Y. Innovative regeneration technology to solve peri-implantitis by Er:YAG laser based on the microbiologic diagnosis: a case series. Int $\mathrm{J}$ Periodontics Restorative Dent35, 67-73 (2015).

21. Eick, S. et al. In Vitro-Activity of Er:YAG Laser in Comparison with other Treatment Modalities on Biofilm Ablation from Implant and Tooth Surfaces. PLoS One12, e0171086 (2017).

22. Takagi, T. et al. Effective removal of calcified deposits on microstructured titanium fixture surfaces of dental implants with erbium lasers. J Periodonto/89, 680-690 (2018).

23. Matsuyama, T., Aoki, A., Oda, S., Yoneyama, T. \& Ishikawa, I. Effects of the Er:YAG laser irradiation on titanium implant materials and contaminated implant abutment surfaces. J Clin Laser Med Surg21, 7-17 (2003).

24. Nejem Wakim, R. et al. Decontamination of Dental Implant Surfaces by the Er:YAG Laser Beam: A Comparative in Vitro Study of Various Protocols. Dentistry Journa/6, 66 (2018).

25. Rupp, F., Scheideler, L., Eichler, M. \& Geis-Gerstorfer, J. Wetting behavior of dental implants. Int J Oral Maxillofac Implants26, 1256-1266 (2011).

26. Zareidoost, A., Yousefpour, M., Ghaseme, B. \& Amanzadeh, A. The relationship of surface roughness and cell response of chemical surface modification of titanium. J Mater Sci Mater Med23, 1479-1488 (2012). 
27. Rupp, F. et al. A review on the wettability of dental implant surfaces I: theoretical and experimental aspects. Acta Biomater10, 2894-2906 (2014).

28. Schneider, L., Laustsen, M., Mandsberg, N. \& Taboryski, R. The Influence of Structure Heights and Opening Angles of Micro- and Nanocones on the Macroscopic Surface Wetting Properties. Sci Rep6, 21400 (2016).

29. Nagy, N. Contact Angle Determination on Hydrophilic and Superhydrophilic Surfaces by Using r-thetaType Capillary Bridges. Langmuir35, 5202-5212 (2019).

30. Parvate, S., Dixit, P. \& Chattopadhyay, S. Superhydrophobic Surfaces: Insights from Theory and Experiment. J Phys Chem B124, 1323-1360 (2020).

31. Lorenzetti, M. et al. The influence of surface modification on bacterial adhesion to titanium-based substrates. ACS Appl Mater Interfaces7, 1644-1651 (2015).

32. Wassmann, T., Kreis, S., Behr, M. \& Buergers, R. The influence of surface texture and wettability on initial bacterial adhesion on titanium and zirconium oxide dental implants. Int $J$ Implant Dent3, 32 (2017).

33. Damante, C.A. et al. In vitro evaluation of adhesion/proliferation of human gingival fibroblasts on demineralized root surfaces by toluidine blue 0 in antimicrobial photodynamic therapy. Photodiagnosis Photodyn Ther13, 303-307 (2016).

34. Ritz, U. et al. The effect of different collagen modifications for titanium and titanium nitrite surfaces on functions of gingival fibroblasts. Clin Oral Investig21, 255-265 (2017).

35. Xu, Z. et al. Enhanced Human Gingival Fibroblast Response and Reduced Porphyromonas gingivalis Adhesion with Titania Nanotubes. Biomed Res Int2020, 5651780 (2020).

36. Yang, Z. et al. Biofunctionalization of zirconia with cell-adhesion peptides via polydopamine crosslinking for soft tissue engineering: effects on the biological behaviors of human gingival fibroblasts and oral bacteria. RSC Advances10, 6200-6212 (2020).

37. Kechagia, J.Z., Ivaska, J. \& Roca-Cusachs, P. Integrins as biomechanical sensors of the microenvironment. Nat Rev Mol Cell Bio/20, 457-473 (2019).

38. Suarez, F., Monje, A., Galindo-Moreno, P. \& Wang, H.-L. Implant surface detoxification: a comprehensive review. Implant Dentistry22, 465-473 (2013).

39. Kearns, V.R., Williams, R.L., Mirvakily, F., Doherty, P.J. \& Martin, N. Guided gingival fibroblast attachment to titanium surfaces: an in vitro study. J Clin Periodonto/40, 99-108 (2013).

40. Atsuta, I. et al. Soft tissue sealing around dental implants based on histological interpretation. $J$ Prosthodont Res60, 3-11 (2016).

41. Mahato, N., Wu, X. \& Wang, L. Management of peri-implantitis: a systematic review, 2010-2015. Springerplus5, 105 (2016).

42. Stanford, C.M. Surface modifications of dental implants. Aust Dent J53 Suppl 1, S26-33 (2008).

43. Marenzi, G. et al. Effect of Different Surface Treatments on Titanium Dental Implant MicroMorphology. Materials (Basel)12 (2019). 
44. Nicolas-Silvente, A.I. et al. Influence of the Titanium Implant Surface Treatment on the Surface Roughness and Chemical Composition. Materials (Basel)13 (2020).

45. Lee, B.S., Shih, K.S., Lai, C.H., Takeuchi, Y. \& Chen, Y.W. Surface property alterations and osteoblast attachment to contaminated titanium surfaces after different surface treatments: An in vitro study. Clin Implant Dent Relat Res20, 583-591 (2018).

\section{Figures}

(a)
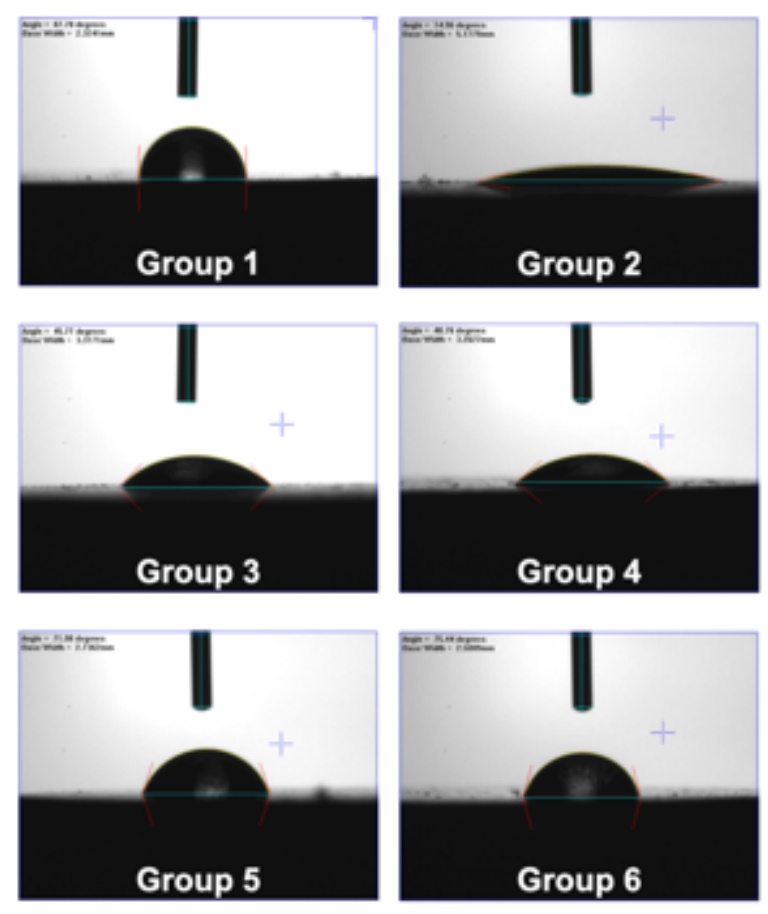

(b)

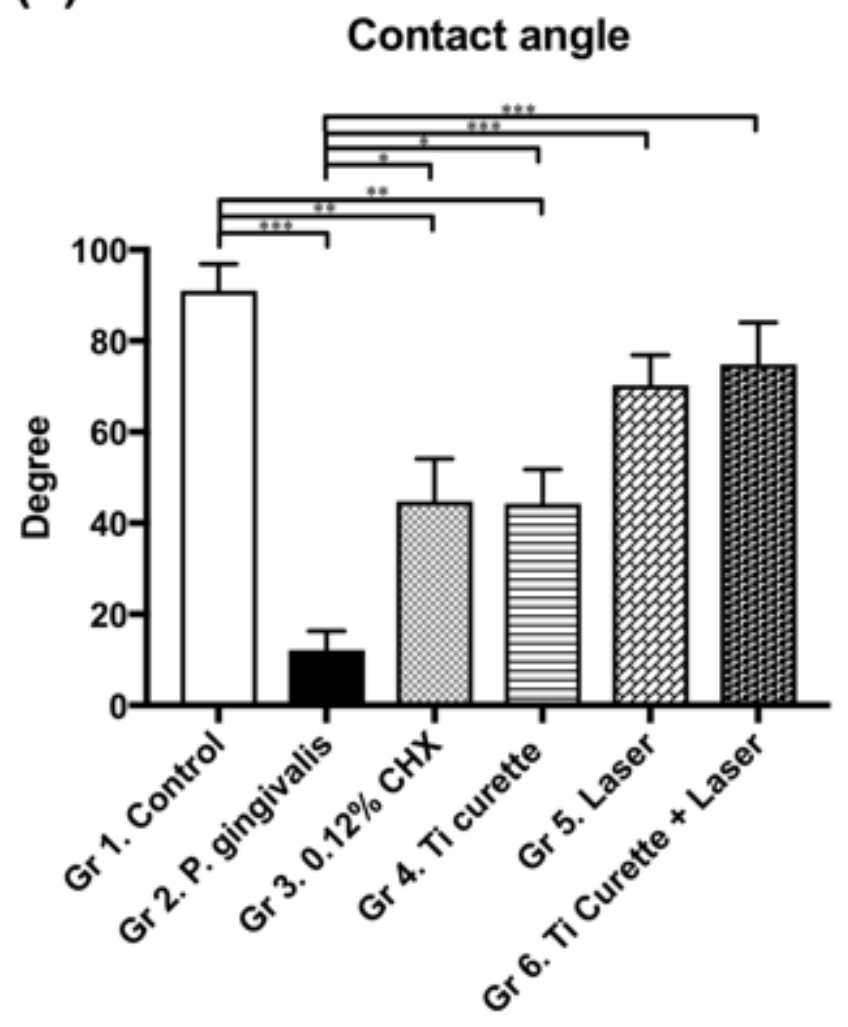

Figure 1

(a) Contact angle photos of P. gingivalis biofilm inoculation on titanium discs after different treatments.

(b) Contact angle of P. gingivalis biofilms after different treatment. ${ }^{*} p<0.05^{* \star} p<0.01{ }^{* \star} p<0.001$ 


\section{Surface roughness}

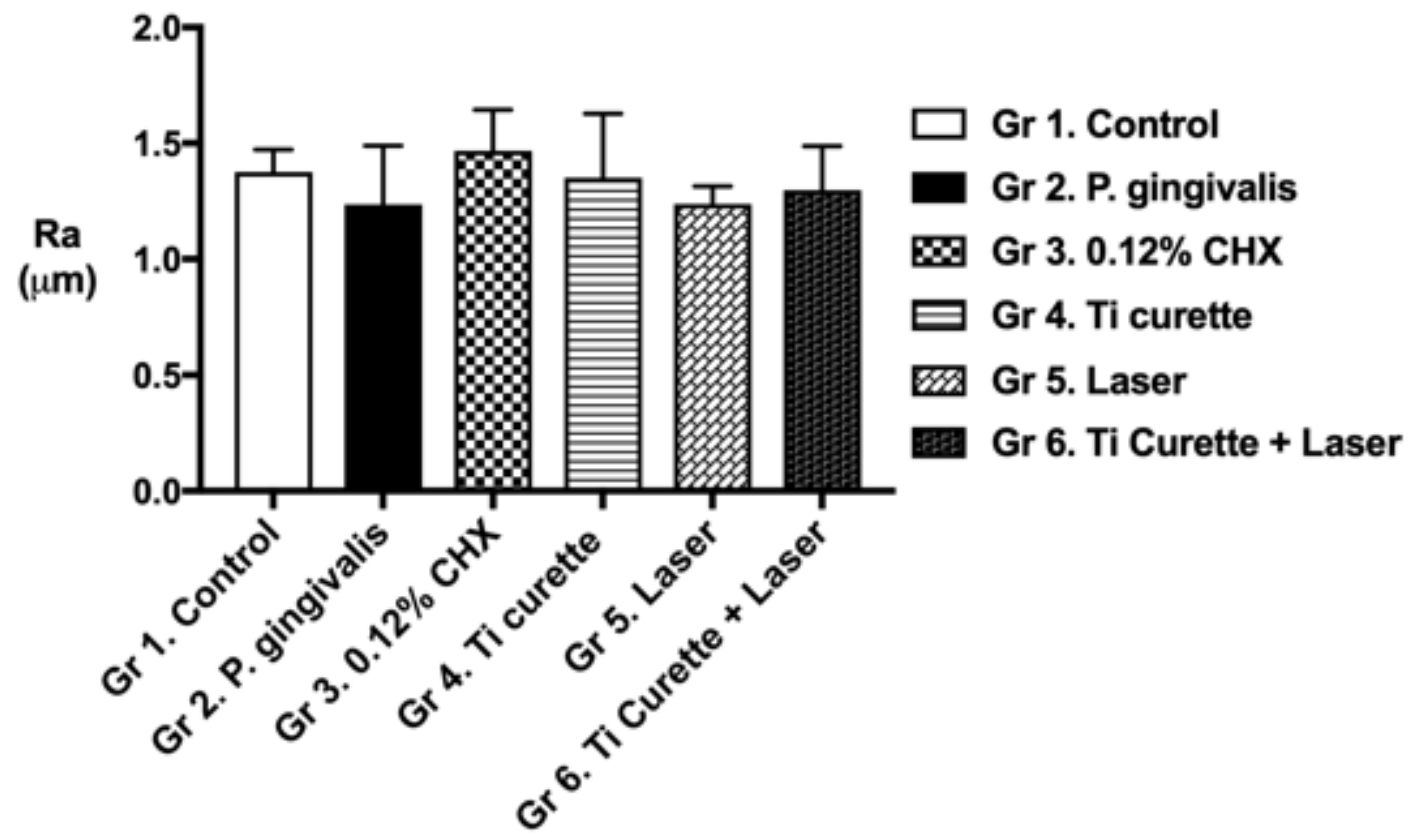

Figure 2

Surface roughness analysis ( $\mathrm{Ra}$ ) of $\mathrm{P}$. gingivalis inoculation on titanium discs after different treatments. ${ }^{*} p<0.05 * * p<0.01 * * * p<0.001$ 


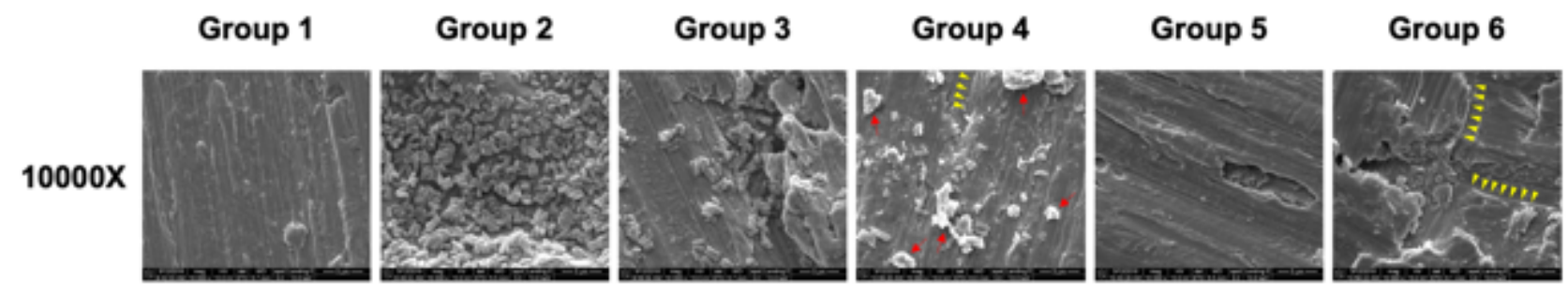

\section{Figure 3}

Scanning electron microscope images of titanium discs after various treatments (Group 1 to Group 6) at magnifications of $\times 10,000$. The red arrow represents titanium particles. The yellow arrow represents scratches caused by curettage. 
(a)

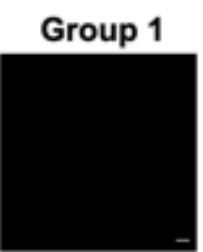

Group 2
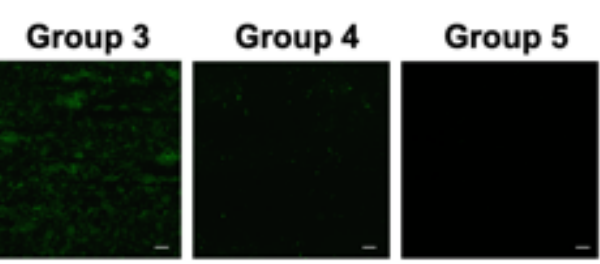

Group 6

SYTO 9

\section{Propidium}

iodide
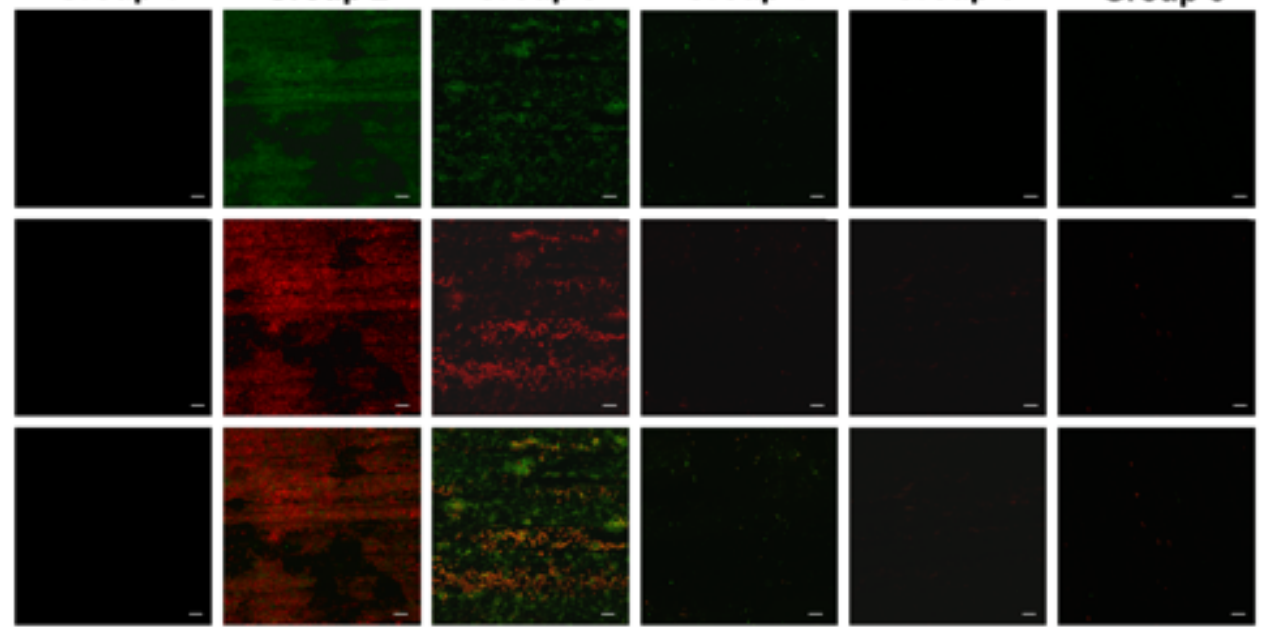

(b)

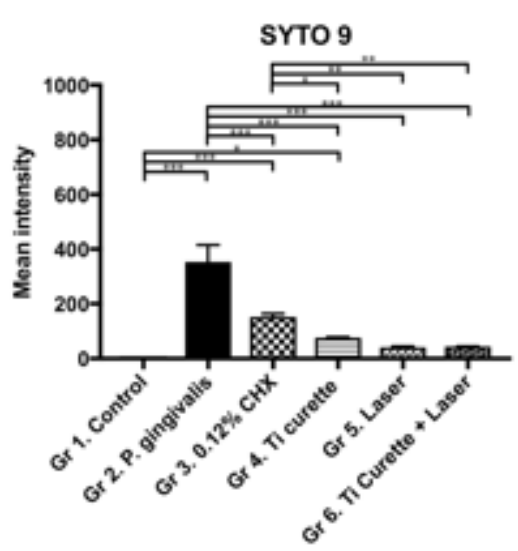

(c)

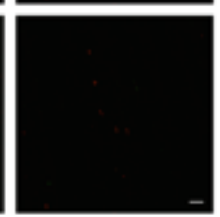

Propidium iodide

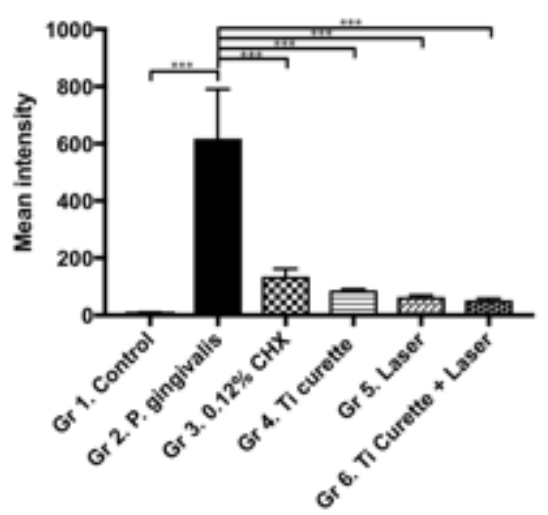

Figure 4

(a) Representative fluorescence microscopy images (×63) with live (green; SYT09)/dead (red; propidium iodide) staining of P. gingivalis ATCC 33277 adhesion on rough titanium disc surfaces after different treatments. Each image was taken with the same $\times 63$ object lens with oil and z-stacked for comparison. Scale bar, $10 \mu \mathrm{m}$ (white). (b) Mean intensity of SYTO 9 and (c) propidium iodide after various treatments. ${ }^{\star} p<0.05 * * p<0.01 * \star * p<0.001$ 
(a)

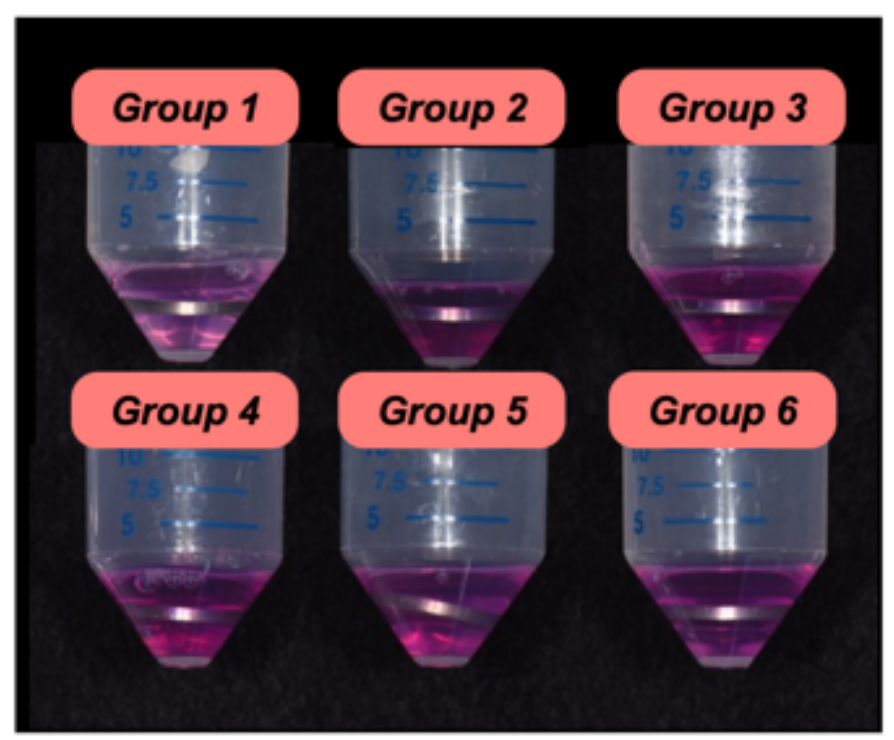

(b)

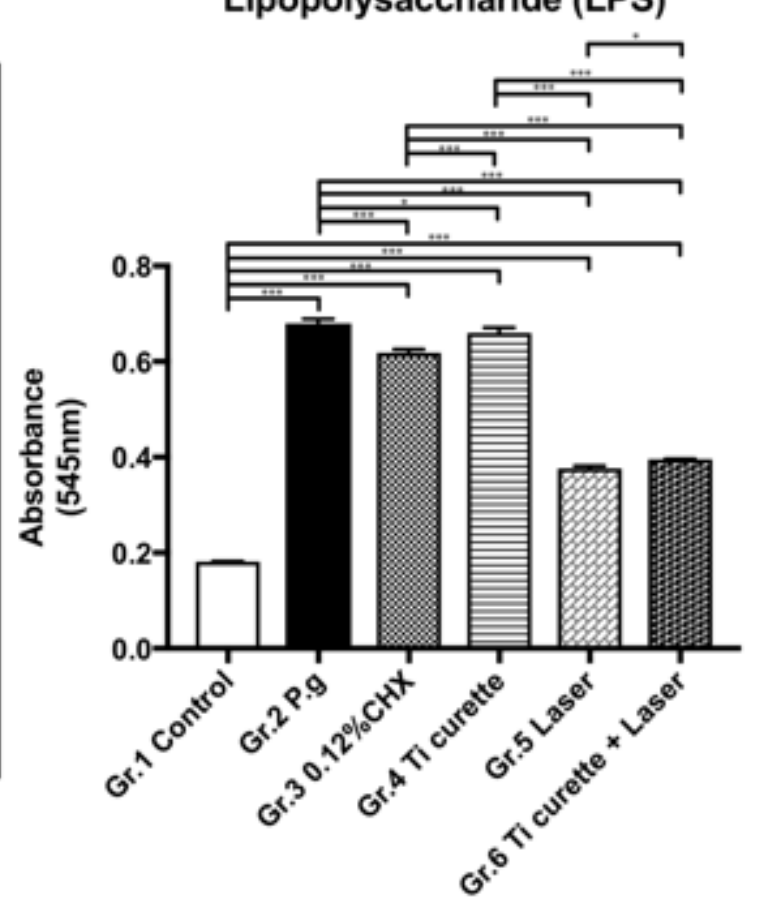

Figure 5

(a) Photos of residual LPS observed during the LAL assay of the titanium discs following different surface treatments. (b) Ultraviolet (UV) absorbance of the residual LPS on titanium discs following various treatments using UV spectrum absorbance at $545 \mathrm{~nm} .{ }^{*} p<0.05 * \star p<0.01 * \star \star p<0.001$ 
(a)
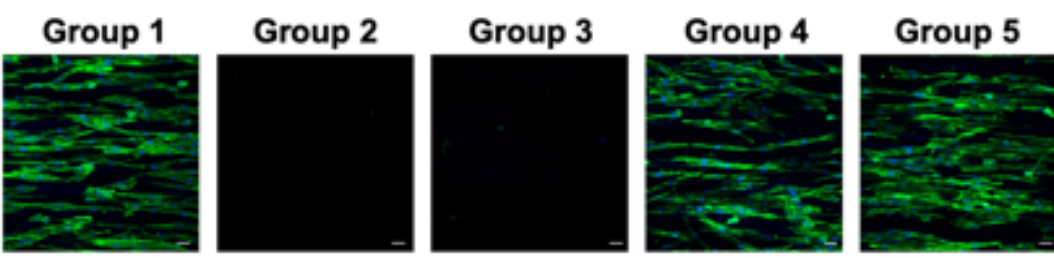

Group 6

24 hours
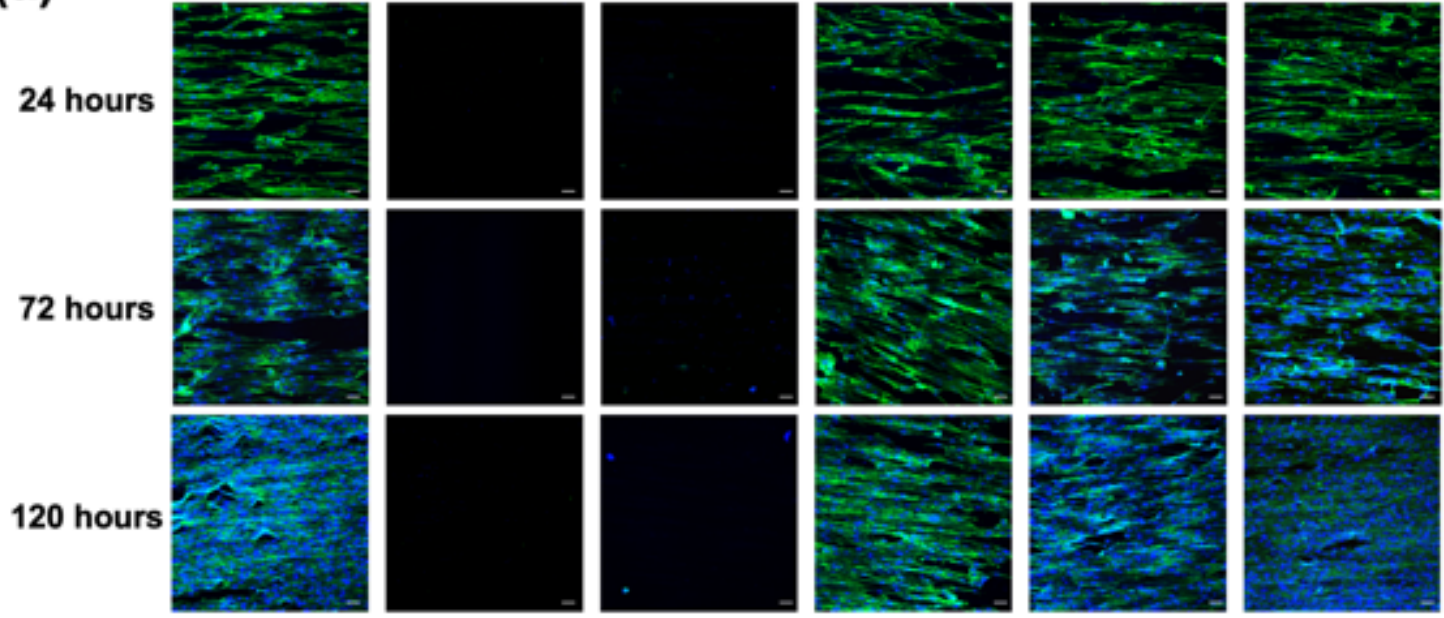

(b)

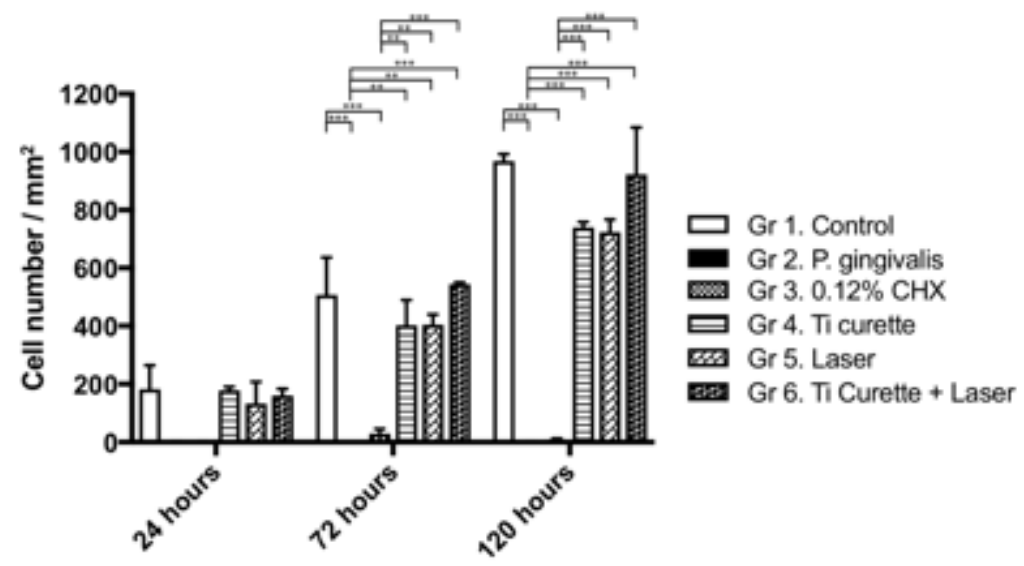

\section{Figure 6}

(a) Representative fluorescence microscopy images (×20) of HGF cells stained with ActinGreenTm 488 (green) indicate a cytoskeleton, and DAPI (blue) was used to label nucleic acids. Each image was taken with the same $\times 20$ object lens and z-stacked for comparison. Scale bar, 50 um (white). (b) HGF cell adhesion assay (cell number per $\mathrm{mm} 2$ ). The cell adhesion number of contaminated titanium discs after different treatments at 24, 72, and 150 h. ${ }^{*} p<0.05{ }^{*} p<0.01{ }^{* *} p<0.001$ 


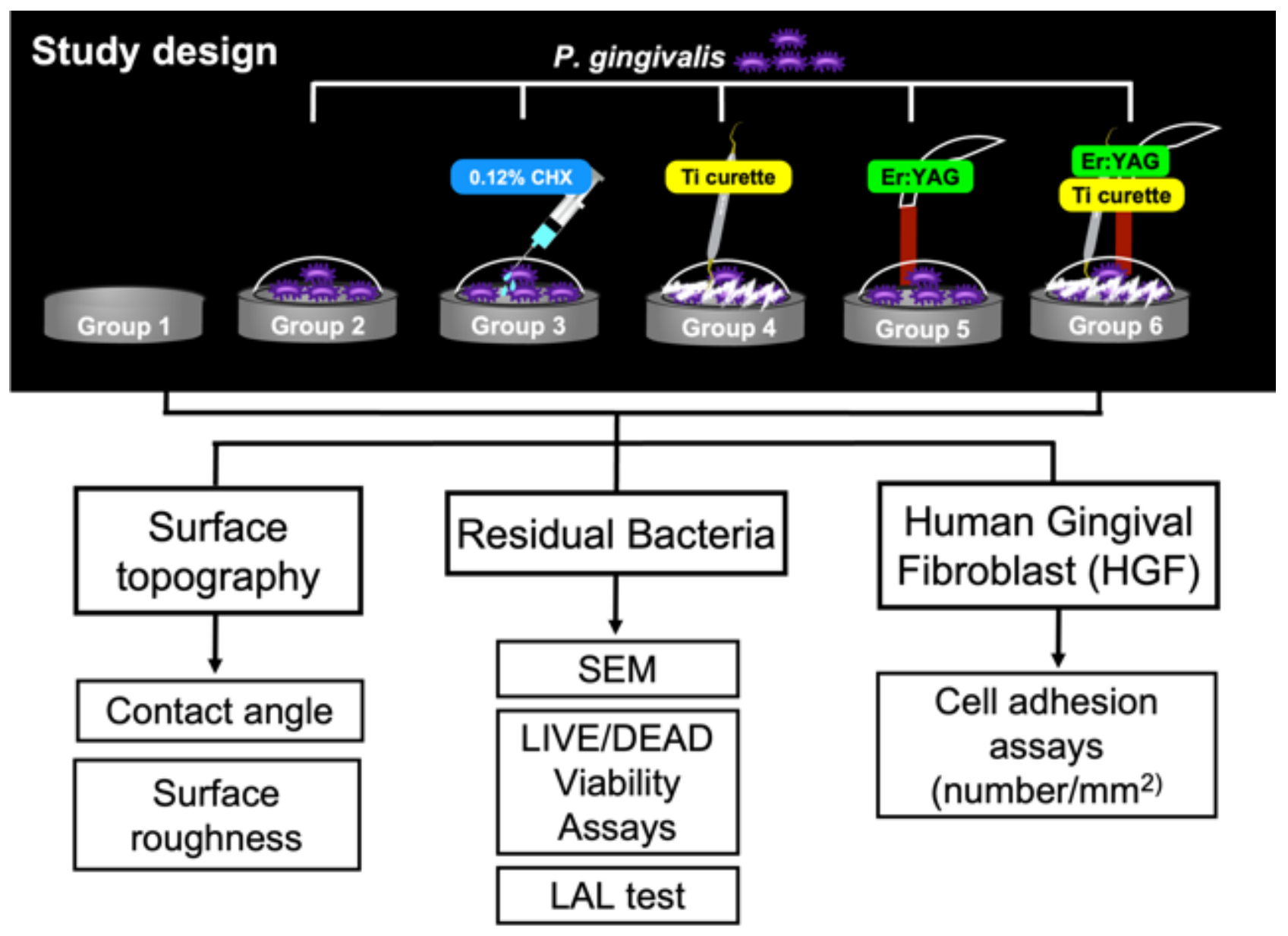

Figure 7

The study design consisted of 3 parts, including P. gingivalis bacterial assessment, an HGF adhesion test, and surface topography analysis. Group 1: negative control; Group 2 to Group 6: P. gingivalis adhesion on titanium discs; Group 2: positive control without treatment; Group 3: treatment with $0.12 \% \mathrm{CHX}$; Group 4 : mechanical debridement with Ti curettage; Group 5: treatment with Er:YAG laser irradiation; Group 6: combined debridement with Ti curettage and Er-YAG laser irradiation. 